\title{
Subacute Thyroiditis - literature overview and COVID-19
}

\section{Remigiusz Domin}

Clinic of Endocrinology, Metabolism and Internal Medicine, Poznan University of Medical Sciences, Poland

(D) https://orcid.org/0000-0002-5190-4056

Corresponding author: remigiusz.domin@gmail.com

\section{Ewelina Szczepanek-Parulska}

Clinic of Endocrinology, Metabolism and Internal Medicine Poznan University of Medical Sciences, 49 Przybyszewskiego Street, 60-555, Poznań, Poland

(iD) https://orcid.org/0000-0003-3661-3274

\section{Daniela Dadej}

Clinic of Endocrinology, Metabolism and Internal Medicine Poznan University of Medical Sciences, 49 Przybyszewskiego Street, 60-555, Poznań, Poland

(iD https://orcid.org/0000-0003-4789-0453

\section{Marek Ruchała}

Clinic of Endocrinology, Metabolism and Internal Medicine Poznan University of Medical Sciences, 49 Przybyszewskiego Street, 60-555, Poznań, Poland

(iD) https://orcid.org/0000-0002-6296-7220
DOI: https://doi.org/10.20883/medical.e472

Keywords: subacute thyroiditis, de quervain thyroiditis, COVIS-19, SARS-CoV-2

Published: 2020-12-29

How to cite: Domin R, Szczepanek-Parulska E, Dadej D, Ruchała M. Subacute Thyroiditis - literature overview and COVID-19. JMS [Internet]. 2020 Dec 29;89(4):e472. doi:10.20883/medical.e472

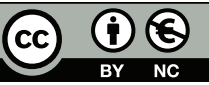

(c) 2020 by the author(s). This is an open access article distributed under the terms and conditions of the Creative Commons Attribution (CC BY-NC) licencse. Published by Poznan University of Medical Sciences

\section{ABSTRACT}

Subacute thyroiditis (SAT), also known as de Quervain's thyroiditis, is a rare thyroid gland disorder, although it is the most common type of painful thyroiditis. The incidence of this disorder is relatively low but likely to be underestimated. Epidemiological studies vary, estimating a prevalence of 12/100,000/year and patients with SAT constitute less than $5 \%$ of all patient consultations due to thyroid disease. For the reason, that SAT can present with a variety of symptoms to different specialists. This review summarises current knowledge of SAT with an emphasis on reports related to SARS-CoV-2.

\section{Introduction}

De Quervain's subacute thyroiditis (SAT) is a rare thyroid gland disorder first described in 1895 by Mygind and later characterised by de Quervain in 1904 [1]. Among thyroid disorders, it is the most common type of painful thyroiditis [2]. It should be highlighted that "de Quervain's thyroiditis" is not synonymous with "de Quervain's disease", a type of tenosynovitis affecting tendons in the first dorsal compartment of the wrist [3]. The incidence of SAT is relatively low, however likely to be underestimated. Due to its diverse and nonspecific symptoms, patients are often diagnosed by family doctors, otolaryngologists, oncologists and other specialists before they eventually consult an endocrinologist. This review summarises the current knowledge of SAT and potential etiological factors (including severe acute respiratory syndrome coronavirus 2 , SARS-CoV-2), clinical presentation, along with present diagnostic and therapeutic options. 


\section{Materials and Methods}

The PubMed search engine was searched for the phrases "subacute thyroiditis", "subacute thyroiditis differential diagnosis", "subacute thyroiditis case report", "thyroiditis differentiation" and "SARS-CoV-2" to identify articles focused on the clinical presentation (including atypical cases), data on etiological factors, novel diagnostic methods and treatment recommendations of SAT.

\section{Subacute thyroiditis}

\section{Epidemiology}

Results of epidemiological studies vary but most indicate that SAT occurs mostly in females, with a peak incidence between 40 and 50 years of age [4]. Its estimated prevalence is equal to $12 / 100000 /$ year, while subjects with SAT constitute less than $5 \%$ of all patients consultations due to thyroid disease. It was also observed that it occurs more frequently among first-degree relatives and twins.

\section{Aetiology}

The aetiology of SAT is unknown but predisposing factors include previous viral infection (i.e. adenoviruses, EBV, Dengue, hepatitis, influenza), immunomodulating treatment or genetic predisposition related to human leukocyte antigens (HLA) $(B * 35, B * 18: 01, D R B 1 * 01$ and $-C * 04: 01)$ [5-9]. HLA-B*35 is the first and the most studied SAT susceptibility gene but its precise role in SAT occurrence is unclear. However, as SAT is related to viral infection, there may be a direct link considering HLA-B*35-restricted viral antigen presentation. There are a few hypotheses on the mechanism. One is molecular mimicry resulting in the cross-reactive immune response - specific viral amino acids may be similar to autologous thyroid peptides, thereby generating autoreactive T-cells that promote an autoimmune response against the thyroid. The second hypothesis is that as a result of infection, changes in tissue components occur through the release of viral products or the presentation of virus antigens by host cells. Then, along with the HLA-B*35 antigen, the thyroid cells are recognised as foreign and destroyed by the immune system [10].

\section{Immunisation-related induction}

\section{COVID-19}

SARS-CoV-2 originated in the Chinese city, Wuhan, in December 2019 and has since become a global pandemic. In most cases, the symptoms are mild, mainly a fever, cough or fatigue but in some people, especially the elderly with comorbidities, it can lead to pneumonia, causing acute respiratory distress syndrome (ARDS) and multiorgan dysfunction (MODS) [11].

The literature search revealed a total of eight patients/cases descriptions ( 7 women and 1 man). Six women aged 18-46 y.o. developed SAT soon after (from a few days up to 6 weeks) recovery from SARS-CoV-2 infection, with typical clinical presentation (i.e anterior neck pain, fever, cough) accompanied by markers of thyrotoxicosis. Only one patient had a previous medical history of thyroid disease (small diffuse non-toxic goitre). Response to treatment with prednisone in five and ibuprofen in one patient was good. On a follow-up visit, the parameters of thyroid function were back to reference ranges in four patients and two patients presented subclinical hypothyroidism [12-14].

Two patients were diagnosed with SAT in the course of SARS-CoV-2 infection. One report concerned a 69-year-old female with a previous history of non-toxic nodular goitre with a dominant benign nodule in the right lobe and repeatedly documented euthyroidism. During recovery after back surgery in hospital, she experienced cough, fever and dyspnoea. A swab confirmed SARS-CoV-2 infection and computed tomography of the chest revealed typical changes related to Coronavirus Disease 19 (COVID-19). On the fifth day of hydroxychloroquine and lopinavir treatment, the patient complained of palpitations, insomnia, and agitation. Thyroid laboratory findings revealed thyrotoxicosis. A thyroid scan using Tc-99 detected no uptake and bedside ultrasonography showed enlarged hypoechogenic goitre with a previously documented nodule in the right lobe. Based on clinical presentation and examination, the patient was diagnosed with SAT, possibly triggered by SARS-CoV-2. Biochemical thyrotoxicosis decreased after treatment with methimazole, intravenous methylprednisolone and oral continuation with prednisolone. Nonsteroid anti-inflammatory drugs (NSAIDs) were 
not implemented because of patients' hypersensitivity. Interestingly, the patient was still positive for SARS-CoV-2 after two months, although the patient was completely asymptomatic [15].

The second report described a 34-year old male primarily diagnosed with COVID-19, who presented new symptoms on the 9th day of illness. He complained of anterior neck pain refractory to paracetamol and dequalinium lozenges and developed tachycardia ranging from 90 to 120 beats/min. No other symptoms were present. Laboratory and ultrasound investigations revealed typical SAT features. After prednisolone and propranolol treatment, his symptoms and thyroid function normalised [16].

\section{Post-vaccine}

The presence of SAT was also documented following vaccines. Passah et al. reported a case of a young female with symptomatic thyrotoxicosis and neck pain of about one-month duration, with no past medical history of infection or sore throat. Eight weeks before symptoms, she was vaccinated with a live influenza virus vaccine. Previously, a few other cases of SAT after vaccination were reported, including the hepatitis B vaccine [17].

Paraplegia, sensory loss, SAT and Dengue fever A 65-year old man was admitted to the Hospital of Guangzhou Medical University (Guangzhou, China) because of acute paraplegia and sensory loss. Almost a week before, he experienced an episode of fever which resolved a few days before admission, with sequential development of neurological symptoms. After laboratory tests and imaging diagnostics, the patient was diagnosed with acute transverse myelitis complicated with SAT as a result of a Dengue viral infection [18].

\section{Clostridium difficile}

SAT is associated with common viral infections. However, a recently published report suggests its induction after bacterial infection. A 24-yearold male soldier admitted for clinical evaluation prior to the military programme, reported mild anterior neck pain, fatigue, night chills and sometimes palpitations persisting for one month. A few months earlier, he was initially treated for Clostridium difficile infection with vancomycin. However, in further investigation, his diarrhoeal problems were still present. Biochemical tests revealed a decreased level of thyroid-stimulating hormone (TSH) and slightly increased T3 and $\mathrm{C}$-reactive protein (CRP), with other thyroid parameters within their reference ranges. Ultrasound examination detected no abnormalities. After evaluation, he was treated with fidaxomicin for 10 days for $C$. difficile infection. He fully recovered and his thyroid parameters went back to reference ranges 8 weeks after the first evaluation [19]. However, in this particular case, in the differential diagnosis, we should consider autoimmune background, as bacterial induction of SAT seems controversial.

\section{Immunomodulating drugs}

The cases presented below document that drugs used as immunomodulatory agents in autoimmune diseases, such as anti-TNF-a inhibitors, can also play a role in the development of SAT [20].

\section{Infliximab}

A 56-year old male suffering from LeśniowskiCrohn Disease started treatment with infliximab to reduce diarrhoea. Four weeks later, he presented with neck pain and swelling with tenderness in the thyroid area. Laboratory tests revealed features of thyrotoxicosis, ultrasound examination demonstrated hypoechogenic goitre and a fine needle biopsy showed amyloid deposition. His symptoms resolved and thyroid function returned to normal after treatment with prednisolone, however, goitre did not. Previously, there were few cases of anti-TNF-a induced SAT but never with accompanying amyloid deposition [21].

\section{Adalimumab}

A 26-year old female suffering from psoriasis and psoriatic arthritis was prescribed adalimumab because of disease progression causing joint degeneration. Seven months later, she started to cough so treatment was discontinued for three weeks, then the patient developed symptoms like fever, sore throat, anterior neck tenderness, lymphadenopathy, hand tremors and palpitations. In laboratory tests, there were features of thyrotoxicosis and hypoechogenic heterogeneous lesions in ultrasound examination. The symptoms resolved, and thyroid function resumed after 
treatment with prednisolone. Due to the increased risk of thyroid diseases in patients receiving antiTNF-a therapy, the authors recommend regular thyroid gland examination before and during treatment with anti-TNF-a inhibitors [22].

\section{Clinical presentation}

\section{Typical}

Inflammation and thyreometabolic dysregulation underlying SAT result in various symptoms, including goitre, pain in the neck area, palpable thyroid nodule or lymphadenopathy. However, other local symptoms like migratory thyroiditis, hard goitre and pain radiation to jaw, ears or chest are also possible. Systemic symptoms are flulike (fever, myalgia, weakness) with acute onset and related to the stage of the disease and thyroid hormones levels range from clinically overt hyper- to hypothyroidism [4, 23-26].

SAT usually comprises consecutive phases of hyper-, hypo- and euthyroid state independently of the initiating factor. The inflammatory process leads to thyroid gland destruction and thyroglobulin $(\mathrm{Tg})$ proteolysis, which results in the release of thyroxine (T4) and triiodothyronine (T3) into the bloodstream. Secondary to that, pituitarythyroid axis regulation is disrupted by the suppression of TSH. With decreasing inflammation, the thyroid regenerates and hormonal homeostasis is restored. Each phase lasts for two to eight weeks [27].

\section{Laboratory findings}

Standard biochemical parameters should be measured including TSH, free thyroid hormones (fT3, fT4), anti-thyroid peroxidase antibodies (aTPO), anti-thyroglobulin antibodies (aTG) and anti-TSH receptor antibodies (TRAb), CRP and erythrocyte sedimentation rate (ESR). In the first phase of SAT, when most diagnoses are made, we should expect biochemical thyrotoxicosis, elevated CRP, elevated ESR and negative thyroid antibodies [28]. However, in some cases, the presence of elevated antibodies (aTPO, aTG, TRAb) may also occur [29].

Hernik et al. investigated the possible use of hepcidin levels in SAT. Hepcidin is a reactive inflammatory protein regulating ferrum homeostasis and is increased during SAT. Promising results show that with a cut-off value, diagnosis is more likely. Additionally, it could be used as a monitoring tool, due to its decreasing manner during effective treatment [28].

An important note is that the diagnosis of SAT cannot be excluded if biochemical parameters are normal. In the study by Tachibana et al., the mean interval between symptoms and abnormal laboratory findings was 6.3 weeks and the longest interval was 11 weeks [30].

\section{Imaging}

Typical imaging diagnostics include ultrasound and scintigraphy, with the use of radioactive iodine and Tc99m. The most common features of ultrasound examination are heterogeneous hypoechoic areas of the affected tissue with a lack of flow on colour Doppler. Most changes are bilateral with characteristic features like "lava flow" [31], in turn, decreasing the uptake of radioactive iodine and Tc99m [32, 33]. Another valuable diagnostic parameter is strain ratio (SR) acquired during real-time sonoelastography of the thyroid gland, which is high in SAT compared to hyperthyroidism and Hashimoto's thyroiditis [34]. Recently, a novel diagnostic tool, a fusion scan of images from ultrasound and 124I-PET was reported, which might be useful when a scintigraphy scan does not match the ultrasound examination findings [35].

Fluorodeoxyglucose Whole-Body PositronEmission Tomography/CT (18F-FDG PET/CT) is another tool. During SAT, there is a possibility of increased FDG uptake, so this condition should be included in the differential diagnosis when assessing lesions with an increased maximum standardised uptake value (SUVmax) [36].

\section{Fine needle aspiration cytology (FNAC)}

The diagnosis of SAT does not require obligatory FNAC examination, though it might be helpful, especially in the exclusion of co-existing thyroid pathologies (i.e. thyroid nodules) and differential diagnosis in cases of uncertainty [37].

\section{Thyroid puzzle - case reports}

\section{Fever of unknown origin}

In 1961, Petersdorf and Beeson characterised fever of unknown origin (FUO) as a body tem- 
perature equal or higher than $38.3^{\circ} \mathrm{C}$ with a duration of three weeks without a diagnosis after one-week intensive inpatient investigation [38]. Currently, due to diagnostic technology development, the one-week inpatient investigation is no longer required but experts suggest certain initial tests to establish the diagnosis of FUO [39]. As SAT can present by fever only, with no other typical clinical signs of thyrotoxicosis, it should be taken into consideration during the evaluation of FUO [2, 40-42].

\section{Acute myocardial infarction and}

\section{sustained ventricular tachycardia}

The most common cause of acute myocardial infarction (AMI) is the rupture of atherosclerotic plaques leading to thrombosis in coronary arteries, which instantly decreases blood flow, that is, the oxygen demand of the myocardium is greater than the oxygen supply. More aetiologies of MI include trauma, vasculitis, drug use, coronary artery anomalies, coronary artery embolism or aortic dissection. Excess demand on the heart, which can be the result of hyperthyroidism, is also listed [43].

Hyperthyroidism/thyrotoxicosis is the first clinical stage of SAT. AMI seems an unlikely presentation of SAT, yet Guerrero et al. reported a case of a 32-year-old female presenting with chest pain and biochemical markers of $\mathrm{MI}$ and thyrotoxicosis with changes in their ECG. There were no disturbances in coronarography and echocardiography. Five days earlier, she was diagnosed with SAT based on neck pain, goitre and fever. The final diagnosis was a thyrotoxic crisis in the course of early relapse of SAT. After treatment with prednisolone and propranolol symptoms, biochemical and ECG changes resolved [44].

Another extremely rare presentation was the case of a 38-year-old woman with no previous medical history, complaining of fatigue and palpitations. Physical examination revealed tender goitre. The ECG showed bigeminy and premature ventricular contractions (PVCs) with a ventricular rate of about $140 \mathrm{bpm}$. During the examination, the monitor displayed an episode of sustained ventricular tachycardia, $278 \mathrm{bpm}$, which was stopped by an infusion of landiolol. Cardiac biochemical parameters were within reference ranges and features of thyrotoxicosis [45].

\section{Encephalopathy}

Encephalopathy is a symptom of brain dysfunction, recognised by an altered mental state and other neurological dysfunctions. Hashimoto encephalitis is also known as "steroid-responsive encephalopathy associated with autoimmune thyroiditis" (SREAT) or "non-vasculitic autoimmune meningoencephalitis" (NAIM) [46]. Chung et al. presented a case of a 49-year-old female admitted to the hospital because of neurological symptoms. Physical examination revealed somnolence, memory impairment, dysarthria, right-hand weakness, gait disturbance and tender goitre. There were no major deviations in laboratory findings besides thyrotoxicosis and brain magnetic resonance imaging was normal. By contrast, imaging of the thyroid presented an acute phase of SAT. After initial treatment with steroids, her mental status improved significantly but two months later, she experienced a relapse in neurological deterioration accompanied by hypothyroidism. Her symptoms resolved after treatment with pulses of methylprednisolone and levothyroxine substitution [47].

\section{Psychosis}

According to the American Psychiatric Association and the World Health Organization, "psychosis" is defined as a state of impaired reality testing with the presence of hallucinations, delusions or both [48]. It can be induced by thyrotoxicosis or hyperthyroidism but generally, psychosis is not the main feature of these conditions. An 18-year-old male presented to the emergency department due to features of psychomotor arousal and paranoid mental state for three days. There was no medical history of previous drug use or psychiatric disorders in the family. Physical examination revealed tachycardia, moist skin, and elevated body temperature, and the thyroid gland was hard and tender on palpation. Laboratory tests revealed biochemical thyrotoxicosis. Due to his mental state, the patient was admitted to the psychiatric department and initially treated with prednisolone and antipsychotic drugs with good effect. On a follow-up visit, the patient remained asymptomatic and was attending school again [49].

\section{Differential diagnosis}

Before setting the diagnosis of the classic variant of SAT, we should take into consideration 
a few differential diagnoses like suppurative thyroiditis [50], Riedel's thyroiditis or haemorrhage to the thyroid nodule. There is also the possibility of a painful variant of Hashimoto's thyroiditis, however, only a dozen cases have been described over the last few decades [51]. Other differential diagnoses should include disorders causing thyrotoxicoses like Graves's disease or intoxication with thyroid hormones medications.

\section{Treatment}

Once the diagnosis is established, treatment should be initiated, however, there is no consensus regarding the scheme of steroid administration. In our department, the following scheme is administered: $40 \mathrm{mg}$ of prednisolone daily in a tapered manner with dose reduction every week for 6-8 weeks. In less severe cases or subjects with contraindications to steroid therapy, the use of NSAIDs like ibuprofen or naproxen can be beneficial, however, is not obligatory. Regarding the study by Sato et al., the effect of stand-alone steroids treatment is superior to NSAIDs [52]. As an additive treatment, in the hyperthyroidism stage, we recommend propranolol and in hypothyroidism levothyroxine but only when the patient is symptomatic.

A novel therapeutic approach was investigated and reported in a study by Shao-Gang et al., intra-thyroid injections of lidocaine and dexamethasone saline solution every other day for one week. The results were compared to typical treatment with oral prednisolone and the injection group was characterised by a more rapid reduction of pain and a shorter duration of treatment [53].

Unfortunately, some patients will need surgical treatment, especially when a malignancy is suspected by FNAC examination or when the patient is unresponsive to pharmacological treatment and symptoms (tender goitre, dysphagia) persist $[54,55]$.

\section{Outcome and recurrence}

Most patients will recover with no long-term complications, though some may experience a relapse or persistent/chronic hypothyroidism.
The recurrence rate is $20-30 \%$, yet the reason for this is still unknown and factors determining the risk of recurrence are lacking. In a recent study by Stasiak et al. focused on haplotypes, the co-presence of HLA-B*18:01 and HLA-B*35 was associated with a higher risk of recurrence, suggesting that high-risk patients should be initially treated with higher doses of steroids with slower dose reduction [56]. Long-lasting hypothyroidism demanding substitution of levothyroxine will occur in $0.5-15 \%$ of patients but predictive factors are still unknown [4].

\section{Final word}

The diagnosis of SAT, also known as de Quervain's thyroiditis, can be challenging due to the diversity in severity and presentation of symptoms. The described inducing factors and various clinical presentations of SAT confirm that this condition is indeed a disease of "thousand faces". Even if recognised as a typical endocrine disorder, due to its insidious nature, ill patients can visit physicians of various specialities, so clinicians should be aware of its symptoms and potential triggering factors.

\section{Acknowledgements}

\section{Conflict of interest statement}

The authors declare no conflict of interest.

\section{Contribution}

R.D. overviewed the literature, prepared draft and did the final editing. E. S-P. is the originator of the paper, collected literature and made major corrections. D.D. proofread the manuscript. M.R. is the senior author. All authors of this paper have read and approved the final version.

\section{Funding sources}

There are no sources of funding to declare.

\section{References}

1. Feinmesser M, Murray D, Colapinto N, Asa SL (1992) Granulomatous and lymphocytic thyroiditis associated with a follicular adenoma. Endocr Pathol 3:105-109. https://doi.org/https://doi.org/10.1007/ BF02921350.

2. Raj R, Yada S, Jacob A, Unnikrishnan D, Ghali W (2018) Fever of unknown origin as a sole presentation of subacute thyroiditis in an elderly patient: A case report with literature review. Case Reports in Endocrinology 2018:e5041724. https://doi. org/10.1155/2018/5041724. 
3. Goel R, Abzug JM (2015) de Quervain's tenosynovitis: a review of the rehabilitative options. Hand 10:1-5. https://doi.org/10.1007/s11552-014-9649-3.

4. Schenke S, Klett R, Braun S, Zimny M (2013) Thyroiditis de Quervain. Nuklearmedizin 52:137-140. https:// doi.org/10.3413/Nukmed-0536-12-10.

5. Abellán Galiana $P$, Pérez-Lázaro A, Aguilera Sancho-Tello V, Merino Torres JF, Berenguer Haym M, Piñón Sellés F (2009) Tiroiditis subaguda inducida por el tratamiento con interferón alfa pegilado y ribavirina en un caso de hepatitis crónica por virus C. Endocrinología y Nutrición 56:136-139. https://doi. org/10.1016/S1575-0922(09)70844-7.

6. Assir MZK, Jawa A, Ahmed HI (2012) Expanded dengue syndrome: subacute thyroiditis and intracerebral haemorrhage. BMC Infectious Diseases 12:240. https://doi.org/10.1186/1471-2334-12-240.

7. Cunha BA, Berbari N (2013) Subacute thyroiditis (de Quervain's) due to influenza A: presenting as fever of unknown origin (FUO). Heart Lung 42:77-78. https:// doi.org/10.1016/j.hrtlng.2012.05.005.

8. Yasuji I (2013) Subacute thyroiditis in a patient with juvenile idiopathic arthritis undergoing etanercept treatment: a case report and review of the literature. Modern Rheumatology 23:397-400. https://doi. org/10.3109/s10165-012-0670-5.

9. Stasiak M, Tymoniuk B, Michalak R, Stasiak B, Kowalski ML, Lewiński A (2020) Subacute thyroiditis is associated with HLA-B*18:01, -DRB1*01 and $-C * 04: 01-$ The significance of the new molecular background. J Clin Med. https://doi.org/10.3390/ jcm9020534

10. Kramer AB, Roozendaal C, Dullaart RPF (2004) Familial occurrence of subacute thyroiditis associated with human leukocyte antigen-B35. Thyroid 14:544547. https://doi.org/10.1089/1050725041517048.

11. Singhal T (2020) A Review of Coronavirus Disease2019 (COVID-19). Indian J Pediatr 87:281-286. https://doi.org/10.1007/s12098-020-03263-6.

12. Brancatella A, Ricci D, Cappellani D, Viola N, Sgrò $D$, Santini F, Latrofa F (2020) Is subacute thyroiditis an underestimated manifestation of SARS-CoV-2 infection? Insights from a case series. J Clin Endocrinol Metab. https://doi.org/10.1210/clinem/dgaa537

13. Brancatella A, Ricci D, Viola N, Sgrò D, Santini F, Latrofa $F(2020)$ Subacute thyroiditis after SarsCOV-2 infection. J Clin Endocrinol Metab. https://doi. org/10.1210/clinem/dgaa276

14. Ruggeri RM, Campennì $A$, Siracusa $M$, Frazzetto G, Gullo D (2020) Subacute thyroiditis in a patient infected with SARS-COV-2: an endocrine complication linked to the COVID-19 pandemic. Hormones 1-3. https://doi.org/10.1007/s42000-02000230-w Ippolito S, Dentali F, Tanda ML (2020) SARSCoV-2: a potential trigger for subacute thyroiditis? Insights from a case report. J Endocrinol Invest 1-2. https://doi.org/10.1007/s40618-020-01312-7.

15. Mattar SAM, Koh SJQ, Rama Chandran S, Cherng BPZ (2020) Subacute thyroiditis associated with COVID19. BMJ Case Rep. https://doi.org/10.1136/bcr-2020237336.
16. Passah A, Arora S, Damle NA, Reddy KS, Khandelwal D, Aggarwal S (2018) Occurrence of subacute thyroiditis following influenza vaccination. Indian $\mathrm{J}$ Endocrinol Metab 22:713-714. https://doi. org/10.4103/ijem.IJEM_237_18.

17. Mo Z, Dong Y, Chen X, Yao H, Zhang B (2016) Acute transverse myelitis and subacute thyroiditis associated with dengue viral infection: A case report and literature review. Exp Ther Med 12:2331-2335. https:// doi.org/10.3892/etm.2016.3604.

18. Mathew J (2018) Clostridium difficile colitis in the setting of subacute thyroiditis: the chicken or the egg. BMJ Case Rep. https://doi.org/10.1136/bcr2018-226711.

19. Surks MI, Sievert R (1995) Drugs and thyroid function. N Engl J Med 333:1688-1694. https://doi. org/10.1056/NEJM199512213332507.

20. Kawashima J, Naoe H, Sasaki Y, Araki E (2015) A rare case showing subacute thyroiditis-like symptoms with amyloid goiter after anti-tumor necrosis factor therapy. Endocrinol Diabetes Metab Case Rep 2015:140117. https://doi.org/10.1530/EDM-14-0117.

21. Wei Y-A, Chuang W-C, Hong C-H (2018) Subacute thyroiditis in a patient with psoriasis treated with a tumor necrosis factor-a inhibitor. Int $\mathrm{J}$ Dermatol 57:869-871. https://doi.org/10.1111/ijd.13941.

22. Salih AM, Kakamad FH, Rawezh QS, Masrur SA, Shvan HM, Hawbash MR, Lhun TH (2017) Subacute thyroiditis causing thyrotoxic crisis; a case report with literature review. Int J Surg Case Rep 33:112-114. https:// doi.org/10.1016/j.ijscr.2017.02.041.

23. Nishihara E, Amino N, Ohye $\mathrm{H}$, Ota $\mathrm{H}$, Ito M, Kubota $\mathrm{S}$, Fukata S, Miyauchi A (2009) Extent of hypoechogenic area in the thyroid is related with thyroid dysfunction after subacute thyroiditis. J Endocrinol Invest 32:3336. https://doi.org/10.1007/BF03345675.

24. Nishihara E, Ohye $H$, Amino N, Takata $K$, Arishima $T$, Kudo T, Ito M, Kubota S, Fukata S, Miyauchi A (2008) Clinical characteristics of 852 patients with subacute thyroiditis before treatment. Intern Med 47:725-729. https://doi.org/10.2169/internalmedicine.47.0740.

25. Sherman SI, Ladenson PW (2007) Subacute thyroiditis causing thyroid storm. Thyroid. https://doi. org/10.1089/thy. 2007.0070

26. Subacute thyroiditis - UpToDate. http://www-1uptodate-1com-1oaxngsms00d5.han.ump.edu.pl/contents/subacute-thyroiditis?search=subacute $\% 20$ thyroiditis\&source=search_result\&selectedTitle= 1 38\&usage_type=default\&display_rank=1\#H6. Accessed 7 Sep 2020

27. Hernik A, Szczepanek-Parulska E, Filipowicz D, Czarnywojtek A, Wrotkowska E, Kramer L, Urbanovych A, Ruchała M (2019) Hepcidin and iron homeostasis in patients with subacute thyroiditis and healthy subjects. Mediators of Inflammation 2019:1-9. https://doi.org/10.1155/2019/5764061.

28. Stasiak M, Lewiński A (2020) Strong correlation between HLA and clinical course of subacute thyroiditis-A report of the three siblings. Genes 11:1282. https://doi.org/10.3390/genes11111282.

29. Tachibana T, Orita $Y$, Ogawara $Y$, Matsuyama $Y$, Abe I, Nakada M, Sato Y, Nishizaki K (2014) Time- 
lag between symptom onset and laboratory findings in patients with subacute thyroiditis. Auris Nasus Larynx 41:369-372. https://doi.org/10.1016/j. anl.2013.11.003

30. Cappelli C, Pirola I, Gandossi E, Formenti A, Agosti B, Castellano M (2014) Ultrasound findings of subacute thyroiditis: a single institution retrospective review. Acta Radiol 55:429-433. https://doi. org/10.1177/0284185113498721

31. Slatosky J, Shipton B, Wahba H (2000) Thyroiditis: Differential diagnosis and management. AFP 61:1047-1052 https://www.aafp.org/afp/2000/0215/ p1047.html

32. Szczepanek-Parulska E, Zybek A, Biczysko M, Majewski P, Ruchała M (2012) What might cause pain in the thyroid gland? Report of a patient with subacute thyroiditis of atypical presentation. Endokrynol Pol 63:138-142. PMID: 22538753

33. Yang Z, Zhang H, Wang K, Cui G, Fu F (2015) Assessment of diffuse thyroid disease by strain ratio in ultrasound elastography. Ultrasound Med Biol 41:2884-2889. https://doi.org/10.1016/j.ultrasmedbio.2015.07.012

34. Freesmeyer M, Opfermann T (2015) Diagnosis of de Quervain's subacute thyroiditis via sensor-navigated 124lodine PET/ultrasound (124I-PET/US) fusion. Endocrine 49:293-295. https://doi.org/10.1007/ s12020-014-0366-z Yeo SH, Lee SK, Hwang I, Ahn EJ (2011) Subacute thyroiditis presenting as a focal lesion on [18F] fluorodeoxyglucose whole-body positron-emission tomography/CT. American Journal of Neuroradiology 32:E58-E60. https://doi.org/10.3174/ ajnr.A2017

35. Vural Ç, Paksoy N, Gök ND, Yazal K (2015) Subacute granulomatous (De Quervain's) thyroiditis: Fine-needle aspiration cytology and ultrasonographic characteristics of 21 cases. Cytojournal 12:9. https://doi. org/10.4103/1742-6413.157479

36. Petersdorf RG, Beeson PB (1961) Fever of unexplained origin: report on 100 cases. Medicine (Baltimore) 40:1-30. https://doi.org/10.1097/00005792196102000-00001

37. Brown I, Finnigan NA (2020) Fever of Unknown Origin (FUO). StatPearls http://www.ncbi.nlm.nih.gov/ books/NBK532265/

38. Koutouridou E, Planck T, Uddman E, Lantz M (2018) Atypical subacute thyroiditis in combination with Grave's disease: Diagnostic difficulties in a case report. Lakartidningen 115: PMID: 29664539

39. Fa B, N W, S H, Ma B (2017) Lesson of the month 1: Subacute thyroiditis: a rare cause of fever of unknown origin. Clin Med (Lond) 17:86-87. https:// doi.org/10.7861/clinmedicine.17-1-86

40. Dalugama C (2018) Asymptomatic thyroiditis presenting as pyrexia of unknown origin: a case report. J Med Case Rep. https://doi.org/10.1186/s13256018-1590-6

41. Mechanic OJ, Grossman SA (2020) Acute Myocardial Infarction. StatPearls http://www.ncbi.nlm.nih.gov/ books/NBK459269/
42. Guerrero E, Loaiz Ortiz D, Manzano D, Tello Montoliu A (2015) Acute myocardial infarction with ST-segment elevation as an early sign of relapse in subacute (De Quervaine) thyroiditis. Emergencias 27:345-346. PMID: 29087065

43. Narita K, Ureshino H, Hashimoto S (2018) Sustained ventricular tachycardia caused by subacute thyroiditis. Intern Med J 48:1160-1162. https://doi. org/10.1111/imj.14018

44. Chong JY, Rowland LP (2006) What's in a NAIM? Hashimoto encephalopathy, steroid-responsive encephalopathy associated with autoimmune thyroiditis, or nonvasculitic autoimmune meningoencephalitis? Arch Neurol 63:175. https://doi. org/10.1001/archneur.63.2.175

45. Chung YJ, Park K-Y, Ahn J, Ha S-Y, Youn YC (2008) Steroid-Responsive recurrent encephalopathy associated with subacute thyroiditis. J Clin Neurol 4:167. https://doi.org/10.3988/jcn.2008.4.4.167

46. Arciniegas DB (2015) Psychosis. Continuum (Minneap Minn) 21:715-736. https://doi.org/10.1212/01. CON.0000466662.89908.e7

47. Subacute thyroiditis presenting as acute psychosis: a case report and literature review. Korean J Intern Med. https://doi.org/10.3904/kjim.2013.28.2.242

48. Dai L, Lin S, Liu D, Wang Q (2020) Acute suppurative thyroiditis with thyroid metastasis from oesophageal cancer. Endokrynologia Polska 71:106-107. https:// doi.org/10.5603/EP.a2019.0053

49. Rotondi M, Capelli V, Locantore P, Pontecorvi A, Chiovato $L$ (2017) Painful Hashimoto's thyroiditis: myth or reality? J Endocrinol Invest 40:815-818. https://doi. org/10.1007/s40618-017-0655-5

50. Sato J, Uchida T, Komiya K, Goto H, Takeno K, Suzuki R, Honda A, Himuro M, Watada H (2017) Comparison of the therapeutic effects of prednisolone and nonsteroidal anti-inflammatory drugs in patients with subacute thyroiditis. Endocrine 55:209-214. https:// doi.org/10.1007/s12020-016-1122-3

51. Ma S-G, Bai F, Cheng $L$ (2014) A novel treatment for subacute thyroiditis: administration of a mixture of lidocaine and dexamethasone using an insulin pen. Mayo Clinic Proceedings 89:861-862. https://doi. org/10.1016/j.mayocp.2014.03.013

52. Mazza E, Quaglino F, Suriani A, Palestini N, Gottero C, Leli R, Taraglio S (2015) Thyroidectomy for painful thyroiditis resistant to steroid treatment: Three new cases with review of the literature. Case Reports in Endocrinology 2015:e138327. https://doi.org/https:// doi.org/10.1155/2015/138327

53. Ranganath R, Shaha MA, Xu B, Migliacci J, Ghossein R, Shaha AR (2016) de Quervain's thyroiditis: A review of experience with surgery. Am J Otolaryngol 37:534537. https://doi.org/10.1016/j.amjoto.2016.08.006

54. Stasiak M, Tymoniuk B, Stasiak B, Lewiński A (2019) The risk of recurrence of subacute thyroiditis is HLADependent. Int J Mol Sci. https://doi.org/10.3390/ ijms20051089 